\title{
ENTROPIC INTEGRATED INFORMATION THEORY -THEORY OF CONSCIOUSNESS
}

\author{
Siddharth Sharma \\ Email:wise.plant.sid@gmail.com
}

\begin{abstract}
In this paper I am going to give a mathematical theory of Integrated Information Theory, using entropy as measure of information and hence, as the information distance function. Also, we will consider a set, whose open subsets are mechanisms and topology is the system, we use these two modification in the structure of Integrated Information Theory [2] and Quantum Integrated Information theory [3], to define Entropic Integrated Information Theory, we will also justify our claims to use why entropy should be use as a measure of cause/effect information and as information distance function using [1]. We will also see the relationship of entanglement with concept, conceptual information and consciousness. This paper is an attempt to binds consciousness, quantum information, entanglement and quantum mechanics together.
\end{abstract}

\section{Introduction}

In this paper we will use the structure of QIIT [3], IIT [2], and taking the cause/effect information distance function as relative entropy to redefine the structure of IIT and QIIT, like cause/effect integrated information, core causes/effects, integrated information of Mechanism, we a set whose topology would be the system and elements of topology would be mechanisms, this will help use to redefine, concept, conceptual structure, conceptual space, conceptual information and integrated information of system or conceptual integrated information, the degree of consciousness. Following is the methology. Also will will observe the relationship between entaglement and consciousness.

\section{$\underline{\text { Structures of Entropic Integrated Information Theory }}$}

The basic structure of Entropic IIT, based on QIIT and IIT are as follows:

- Structure I: Let $\Lambda$ be a set with cardinality $|\Lambda|<\infty$ and topology $\mathcal{T}$. Here elements of the topology is mechenisms and topology itself is the system For each $i \in \Lambda$ there is an associated $d$-dimensional quantum system with Hilbert space $\mathbb{H}_{i} \cong \mathbb{C}^{d}$. Given any $\Omega \in \mathcal{S} \subset \mathcal{T}$ we define $\mathbb{H}_{\Omega}:=\bigotimes_{i \in \Omega} \mathbb{H}_{i}$, with dimension $d^{|\Omega|}$.

- $\quad$ Structure II: The state-space is the associated operator algebra denoted by $L\left(\mathbb{H}_{\Lambda}\right)$. 
- $\quad$ Structure III: One has that $\mathbb{H}_{\Lambda}:=\mathbb{H}_{\Omega} \otimes \mathbb{H}_{\Omega^{\prime}}$, where $\Omega^{\prime}$ denotes the complement of $\Omega \in$ $\mathcal{S} \subset \mathcal{T}$.

- Structure IV: Let the dynamics be described by a unitary completely positive-map $\mathcal{U}_{\tau}: L\left(\mathbb{H}_{\Lambda}\right) \rightarrow L\left(\mathbb{H}_{\Lambda}\right)$, such that $\mathcal{U}_{\tau}\left(\Psi_{\Lambda}(t)\right):=\Psi_{\Lambda}(t+\tau)$ with $\mathcal{U}(\mathbb{I})=\mathbb{I}_{\Lambda}$, where $\mathbb{I}_{\Lambda}$ is identity of operator $\mathbb{H}_{\Lambda}$.

- Structure $\mathbf{V}$ : Given $\Omega \in \mathcal{S} \subset \mathcal{T}$ we define the noising completely positive-map $\mathcal{N}_{\Omega}$ by $\mathcal{N}_{\Omega}: L\left(\mathbb{H}_{\Lambda}\right) \rightarrow L\left(\mathbb{H}_{\Lambda}\right)$, such that $\mathcal{N}_{\Omega}\left(\Psi_{\Lambda}\right):=\left(\operatorname{tr}_{\Omega} \Psi_{\Lambda}\right) \otimes \frac{\mathbb{I}_{\Omega}}{d^{|\Omega|}}$

- Structure VI: let $\rho, \sigma$ be density matrices, then $\mathcal{D}[\rho, \sigma]$ is the information distance function and defined as $\mathcal{D}[\rho, \sigma]:=|S(\rho|| \sigma)|$ the modulus of relative entropy (e.g. relative von neumann entropy, renyi entropy), which is obvious as entropy is measure of information.[5]

We know that the relative entropy is not symmetric function but Johannes Kleiner and Sean Tull, clearly mention in the paper [1] that

".. the distance function does not necessarily have to satisfy the axioms of a metric. While ......natural axioms.....might hold, they are not necessary for the IIT algorithm."

Further structures require a detained information and hence are explained in their own sections below.

\section{$\underline{\text { Cause/Effect Repertoires }}$}

- Definition: Given the unitary $\mathcal{U}_{\tau}$, the state $\Psi_{\Lambda} \in L\left(\mathbb{H}_{\Lambda}\right),\left(\Psi_{\Lambda} \geq 0, \operatorname{tr}\left(\Psi_{\Lambda}\right)=1\right)$ and the mechanisms $M, P \in \mathcal{S} \subset \mathcal{T}$ we define the effect $(e)$ and cause $(c)$ repertoire of $M$ over the purview $P$, by:

$$
\rho^{(x)}(P \mid M):=\operatorname{tr}_{P^{\prime}}\left(\mathcal{U}_{\tau}^{(x)} \circ \mathcal{N}_{M^{\prime}}\left(\Psi_{\Lambda}\right)\right)
$$

Where $x \in\{e, c\}$. Let $\mathcal{U}_{\tau}^{(e)}=\mathcal{U}_{\tau}$ then $\mathcal{U}_{\tau}^{(c)}=\mathcal{U}_{\tau}^{*}$, the dual of $\mathcal{u}$.

- The set of density matrices $\rho^{(e)}(P \mid M)\left(\rho^{(c)}(P \mid M)\right)$ encode how the dynamics constrain the future (past) of $P$, given that the system is initialized in $\Psi_{\mathrm{M}}$ and noised over $M^{\prime}$.

- Note, $\rho^{(e)}(\varnothing \mid M)=\mathbb{I}=\rho^{(c)}(\varnothing \mid M), \forall M$ by trace normalization 


\section{Cause/Effect Information}

- Definition: The cause/effect information, $\mathcal{J}_{x}(P \mid M)$ of $M$ over $P$ is given by

$$
\mathcal{J}_{x}(P \mid M):=\mathcal{D}\left[\rho^{(x)}(P \mid M), \rho^{(x)}(P \mid \emptyset)\right]
$$

where it is easy to evaluate $\rho^{(x)}(P \mid \emptyset):=\frac{\mathbb{I}_{\mathrm{P}}}{d^{|P|}}$

- distance monotonicity under partial traces and unitary invariance follows $O \in \mathcal{T}_{P} \Rightarrow$ $\mathcal{J}_{x}(O \mid M) \leq \mathcal{J}_{x}(P \mid M)$, where $x \in\{e, c\}$.

\section{Cause/Effect Integrated Information}

- Given the mechanism $M$ and the purview $P$ we consider all possible bi-partitions of them $\left\{M_{i}, \widetilde{M}_{i}\right\}$ and $\left\{P_{i}, \tilde{P}_{i}\right\}$, where $X_{i} \cap \tilde{X}_{i}=\varnothing, X_{i} \cup \tilde{X}_{i}=X$ and $X_{i}, \tilde{X}_{i} \in \mathcal{T}_{X}$. Where $\mathcal{T}_{X}$ is sub topology of $X$ and $X \in\{M, P\}$.

- Definition: cause/effect integrated information of $M$ over $P$ by

$$
\varphi^{(x)}(P \mid M):=\min _{i} \mathcal{D}\left[\rho^{(x)}(P \mid M), \rho^{(x)}\left(P_{i} \mid M_{i}\right) \otimes \rho^{(x)}\left(\tilde{P}_{i} \mid \widetilde{M}_{i}\right)\right]
$$

where $x \in\{e, c\}$. In this definition the minimum is taken over all the $2^{|P|+|M|-1}-1$ possible pairings $\left(P_{i}, M_{i}\right)$ different from the trivial one $(\varnothing, \varnothing),(P, M)$, which would make any repertoire factorizable.

- The e relative entropy of entanglement, $E_{R E}^{(x)}(P \mid M)$ which is the measure of entanglement in the density matrix $\rho^{(x)}(P \mid M)$ is the lower bound of the cause/effect integrated information and it's upper bound is cause/effect information.

$$
E_{R E}^{(x)}(P \mid M) \leq \varphi^{(x)}(P \mid M) \leq \mathcal{J}_{x}(P \mid M)
$$

where

$$
E_{R E}^{(x)}(P \mid M):=\min \left\{S\left(\rho^{(x)}(P \mid M) \| \sigma_{i} \otimes \widetilde{\sigma}_{l}\right)\right\}
$$

Also, $\sigma_{i} \in L\left(\mathbb{H}_{i}\right), \widetilde{\sigma}_{l} \in L\left(\widetilde{\mathbb{H}}_{i}\right)$ and $\mathbb{H}_{i} \otimes \widetilde{\mathbb{H}}_{i}=\mathbb{H}_{P}, \mathbb{H}_{i}, \widetilde{\mathbb{H}}_{i} \neq \mathbb{H}_{P}$ with each $\sigma_{i}$ and $\widetilde{\sigma}_{\imath}$ are positive definite and has trace equals unity.

\section{Core Causes/Effects}

- Definition: The purview 


$$
P_{\max }^{(x)}:=\left\{P \mid \max _{P \in \mathcal{S}} \varphi^{(x)}(P \mid M)\right\}
$$

is defined a core effect/cause of $M$, where $x \in\{e, c\}$.

- The corresponding value of $\varphi^{(x)}$ is denoted by

$$
\varphi^{(x)}(M):=\max _{P \in \mathcal{S}} \varphi^{(x)}(P \mid M)=\varphi^{(x)}\left(P_{\max }^{(x)} \mid M\right)
$$

where $x \in\{e, c\}$.

- The associated repertoires are called global given by

$$
\rho_{\varphi}^{(x)}(M):=\rho^{(x)}\left(P_{\max }^{(x)} \mid M\right) \otimes \frac{\mathbb{I}_{Q_{\max }^{(x)}}}{d^{\left|Q_{\max }^{(x)}\right|}}
$$

Where $Q_{\max }^{(x)}$ complement $P_{\max }^{(x)}$, i.e. $Q_{\max }^{(x)}:=\Lambda \backslash P_{\max }^{(x)}$, where $x \in\{e, c\}$

- The corresponding value of $E_{R E}^{(x)}$ is denoted by

$$
E_{R E}^{(x)}(M):=\max _{P \in \mathcal{S}} E_{R E}^{(x)}(P \mid M)=E_{R E}^{(x)}\left(P_{\max }^{(x)} \mid M\right)
$$

where $x \in\{e, c\}$

\section{Integrated Information of Mechanism}

- The integrated information of $M$ is defined as

$$
\varphi(M):=\min \left\{\varphi^{(e)}(M), \varphi^{(c)}(M)\right\}
$$

- If $\varphi(M)=0$ then either $\varphi^{(e)}(P \mid M)=0, \forall P$ or $\varphi^{(c)}(P \mid M)=0, \forall P$. If, $\varphi(M)>0$ then it creates a concept.

- We will define integrated entanglement information as

$$
E_{R E}(M):=\min \left\{E_{R E}^{(e)}(M), E_{R E}^{(c)}(M)\right\}
$$

- It is interesting to note that since, entanglement entropy is the lower bound, hence if there is entanglement in quantum system then that would lead to a concept 


\section{Conceptual Structure}

- For any mechanism $M \in \mathcal{S}$ the triple $\left(\varphi(M) ; \rho_{\varphi}^{(e)}(M), \rho_{\varphi}^{(c)}(M)\right)$ with $\varphi(M)>0$ is called a concept associated with $\varphi(M)$, denoted by $C_{\mathcal{S}}(M)$. The conceptual structure, $\mathcal{C}_{\mathcal{S}}$ is defined as follows i.e.

$$
\mathcal{C}_{\mathcal{S}}:=\left(C_{\mathcal{S}}(M) \mid \varphi(M) \text { is the integrated information of } M\right)_{M \in \mathcal{S}}
$$

Where $\mathcal{S} \subset \mathcal{T}$ is the subset of topology, $\mathcal{T}$ of set $\Lambda$.

\section{Integrated Information of System}

- Conceptual space is metric space of conceptual structure. metric $\Delta$ defined as, given two conceptual structures $\mathcal{C}_{\mathcal{S}}$ and $\mathcal{C}_{\mathcal{R}}$, where $\mathcal{S}, \mathcal{R} \subset \mathcal{T}$

$$
\Delta\left(\mathcal{C}_{S}, \mathcal{C}_{\mathcal{R}}\right):=\left\|\mathcal{C}_{\mathcal{S}}\right\|-\left\|\mathcal{C}_{\mathcal{R}}\right\| \mid
$$

Where

$$
\left\|\mathcal{C}_{\mathcal{S}}\right\|:=\sum_{\substack{x \in\{e, \mathcal{C}\} \\ M \in \mathcal{S}}} \varphi(M)\left|S\left(\rho_{\varphi}^{(x)}(M)\right)\right|
$$

is the conceptual information and $S\left(\rho_{\varphi}^{(x)}(M)\right)$ is entropy of density matrix $\rho_{\varphi}^{(x)}(M)$.

- It is again interesting two see that if we replace $E_{R E}(M)$ in place of $\varphi$ it serves as a lower bound to conceptual information, hence we define conceptual entanglement information as,

$$
\left\|\mathcal{C}_{\mathcal{S}}\right\|_{E_{R E}}:=\sum_{\substack{x \in\{e, c\} \\ M \in \mathcal{S}}} E_{R E}(M)\left|S\left(\rho_{\varphi}^{(x)}(M)\right)\right|
$$

Which serves as the lower bound to conceptual information

- Giulio Tononi defines Integrated conceptual information $(\Phi)$ in his final paper on IIT as "Conceptual information that is generated by a system above and beyond the conceptual information generated by its (minimal) parts......"

- Considering system as topology $\mathcal{T}$ we define

$$
\Phi:=\min _{\substack{\mathcal{S} \subset \mathcal{T} \\ \mathcal{S} \neq \mathcal{T}}} \Delta\left(\mathcal{C}_{\mathcal{T}}, \mathcal{C}_{\mathcal{S}}\right)
$$


if we replace $\varphi(M)$ with $E_{R E}(M)$ in $\Phi$ ( lets call it $\Phi_{E_{R E}}$ ) then $\Phi_{E_{R E}} \leq \Phi$, hence if entegalment occurs it causes consciousness.

\section{Disscusion and Conslusion}

This theory helps to understand the relationship between entropy and consciousness, it also suggests that entanglement can cause cansioness. This explains the breakdown of wave function due to entanglement. This theory can help us to understand how quantum phenomena leads to classical reality at large scale and why quantum phenomena are not generally observed at large scale thereby generating a relationship between classical and quantum physics.

\section{$\underline{\text { References }}$}

1. The Mathematical Structure of Integrated Information Theory Johannes Kleiner, Sean Tull arXiv:2002.07655 [q-bio.NC]

2. Oizumi, M., Albantakis, L., \& Tononi, G. (2014). From the Phenomenology to the Mechanisms of Consciousness: Integrated Information Theory 3.0. PLoS Computational Biology, 10(5). https://doi.org/10.1371/journal.pcbi.1003588

3. Zanardi, Paolo \& Tomka, Michael \& Campos Venuti, Lorenzo. (2018). Quantum Integrated Information Theory.

4. Petz D. (2008) Prerequisites from Quantum Mechanics. In: Quantum Information Theory and Quantum Statistics. Theoretical and Mathematical Physics. Springer, Berlin, Heidelberg. https://doi.org/10.1007/978-3-540-74636-2_2

5. Petz D. (2008) Information and its Measures. In: Quantum Information Theory and Quantum Statistics. Theoretical and Mathematical Physics. Springer, Berlin, Heidelberg. https://doi.org/10.1007/978-3-540-74636-2_3

6. Petz D. (2008) Entanglement. In: Quantum Information Theory and Quantum Statistics. Theoretical and Mathematical Physics. Springer, Berlin, Heidelberg. https://doi.org/10.1007/978-3-540-74636-2_4

7. Sharma, S. (2020, December 5). QUANTUM INFORMATION, ENTANGLEMENT AND ENTROPY. https://doi.org/10.31219/osf.io/a3whf

8. Kleiner, J. Mathematical Models of Consciousness. Entropy 2020, 22, 609.

9. Bengtsson, I., \& Zyczkowski, K. (2006). Geometry of Quantum States: An Introduction to Quantum Entanglement. Cambridge: Cambridge University Press. doi:10.1017/CBO9780511535048 
10. Watrous, J. (2018). The Theory of Quantum Information. Cambridge: Cambridge University Press. doi:10.1017/9781316848142

11. G. Tononi, BMC Neuroscience 5, 42 (2004).

12. M. Oizumi, L. Albantakis, and G. Tononi, PLOS Computational Biology 10, 5 (2014)

13. K. Kremnizer and A. Ranchin, Found. Phys. 45, 889 (2015).

14. L. Albantakis and G. Tononi, Entropy 17, 5472 (2015).

15. M. Tegmark, Chaos, Solitons \& Fractals 76, 238 (2015).

16. M. Tegmark, PLOS Computational Biology 12, 11 (2016).

17. G. Tononi and C. Koch, Phil. Trans. R. Soc. B 370, 20140167 (2015)

18. E. Lieb, D. Robinson, Commun. Math. Phys. 28, 251 (1972)

19. Jaeger G. (2009) Superposition, Entanglement, and Limits of Local Causality. In: Entanglement, Information, and the Interpretation of Quantum Mechanics. The Frontiers Collection. Springer, Berlin, Heidelberg. https://doi.org/10.1007/978-3-540$\underline{92128-8 \_1}$

20. Jaeger G. (2009) Quantum Measurement, Probability, and Logic. In: Entanglement, Information, and the Interpretation of Quantum Mechanics. The Frontiers Collection. Sp Jaeger G. (2009) Interpretations of Quantum Mechanics. In: Entanglement, Information, and the Interpretation of Quantum Mechanics. The Frontiers Collection. Springer, Berlin, Heidelberg. https://doi.org/10.1007/978-3-540-92128-8_3 ringer, Berlin, Heidelberg. https://doi.org/10.1007/978-3-540-92128-8_2

21. Jaeger G. (2009) Interpretations of Quantum Mechanics. In: Entanglement, Information, and the Interpretation of Quantum Mechanics. The Frontiers Collection. Springer, Berlin, Heidelberg. https://doi.org/10.1007/978-3-540-92128-8_3

22. Jaeger G. (2009) Information and Quantum Mechanics. In: Entanglement, Information, and the Interpretation of Quantum Mechanics. The Frontiers Collection. Springer, Berlin, Heidelberg. https://doi.org/10.1007/978-3-540-92128-8_4 